\title{
The use of Tranexamic Acid (TXA) Among Obstetricians and Gynecologists: A pilot study of current clinical practice.
}

\author{
Rebecka Bogue Docken ${ }^{1}$, Mary Lynn ${ }^{1}$, Sereen Nashif ${ }^{1}$, Callie Hurtt ${ }^{1}$, Lindsey McAlarnen ${ }^{1}$, \\ and jean Goodman ${ }^{1}$ \\ ${ }^{1}$ Loyola University Medical Center
}

May 20, 2020

\begin{abstract}
Objective: To investigate current practice patterns of tranexamic acid (TXA) use by Obstetricians and Gynecologists. Design: A cross-sectional, web based survey. Population: Members of the Central Association of Obstetricians and Gynecologists (CAOG). Methods: The anonymous survey regarding demographics, circumstances of use, and barriers of TXA use was emailed to CAOG members October 2018 - December 2018. Results: There were 81 responses with a response rate of $27 \%$. The majority of respondents were general obstetricians and gynecologists (63\%). TXA used mostly during postpartum hemorrhage (72\%), followed by heavy cyclic bleeding (42\%), and then benign gynecological surgeries (5\%). The majority of participants had TXA as part of their hospitals hemorrhage protocol $(73 \%)$ and increase use was associated with having a hospital protocol (86\%). The largest concern with TXA use was thromboembolic events (48\%). The majority of respondents had a favorable view of TXA being incorporated into hospital protocols (95\%). Conclusion: To our knowledge this is the first survey among obstetricians and gynecologist to elicit current practice patterns with TXA. This study illustrates an association between increase use of TXA when it is incorporated into a hospital protocol for postpartum hemorrhage. This finding suggests that to an individual provider, local hospital recognition of the safety and effectiveness of TXA is more impactful and influential than the World Health Organization to an individual obstetrician in practice. The major concern with TXA use was increased risk of a thromboembolic event, an overwhelming number of respondents would support incorporating TXA into their hospital's protocols
\end{abstract}

\section{INTRODUCTION}

Bleeding is a recognized complication of both obstetrical and gynecological procedures. In fact, obstetrical hemorrhage, a major cause of maternal morbidity ${ }^{1}$ is the leading cause of maternal death worldwide ${ }^{2}$. In gynecology, bleeding during benign gynecological surgery and due to benign gynecologic conditions is a major cause of morbidity ${ }^{3}$. Numerous studies show that tranexamic acid (TXA) decreases blood loss effectively in general surgical procedures; especially in trauma surgery ${ }^{4,5,6,7}$. The WOMAN trial demonstrates that TXA use for postpartum hemorrhage (PPH) reduces mortality. When used for postpartum hemorrhage, it causes no adverse events and most notably, no thromboembolic events ${ }^{8}$. The World Health Organization (WHO) recommends considering TXA in the standard postpartum hemorrhage protocol for both vaginal and cesarean sections ${ }^{9}$.

Currently, in the United States, cesarean sections account for $32 \%$ of all deliveries ${ }^{10}$. The blood loss for a cesarean delivery averages between $800 \mathrm{ml}$ to $1000 \mathrm{ml}^{11}$. Approximately $5 \%$ of cesarean sections and $1 \%$ of vaginal deliveries require a blood transfusion ${ }^{12}$. In 2018, births in the United States totaled 3,791,712 ${ }^{13}$. TXA has been shown to be an effective medication to decrease bleeding during cesarean section not only when used acutely but also prophylactically ${ }^{14,15,16}$.

TXA has been used since the 1970s as a non-hormonal medication to decrease menstrual bleeding in women 
with menorrhagia ${ }^{17}$. When compared to placebo it decreases heavy menses by $40 \%^{18}$. More recently, TXA use has been expanded to include benign surgical procedures such as hysterectomy and myomectomy. Hysterectomy is one of the most commonly performed surgical procedures in the United States, approximately 600,000 are performed each year. It is estimated that one in nine women will have a hysterectomy in their lifetime ${ }^{19}$. In recent years, randomized control trials and meta-analysis investigating TXA use in benign gynecology, for both hysterectomies and myomectomies, show an overall reduction in both total blood loss and need for reoperations due to postoperative hemorrhage $e^{20,21,22}$.

One commonality of these studies on TXA use is the lack of adverse events. Venous thromboembolism with the use of TXA for PPH, heavy menstrual bleeding, or benign gynecologic surgery is not shown to be a concern. Given these findings and the World Health Organization's recommendations, our goal in conducting this study was to achieve a better understanding of the current use practices of TXA among obstetricians and gynecologists.

\section{METHODS}

We conducted a cross sectional online survey questionnaire. Providers who were members of the Central Association of Obstetricians and Gynecologists (CAOG) were invited to participate in the online survey. In determining a provider population to survey we chose CAOG because its member population represents the 'central' 29 states. These include Alabama, Arizona, Arkansas, Colorado, Idaho, Illinois, Indiana, Iowa, Kansas, Kentucky, Louisiana, Michigan, Minnesota, Mississippi, Missouri, Montana, Nebraska, Nevada, New Mexico, North Dakota, Ohio, Oklahoma, South Dakota, Tennessee, Texas, Utah, West Virginia, Wisconsin, and Wyoming. This is a nonprofit organization of physicians and is one of the oldest organizations, founded in $1951^{23}$.

The survey was sent initially to members in October 2018 and two subsequent requests for completion were sent out in November 2018 and December 2018. The survey was closed in January 2019. The survey comprised of 10 questions. These questions specifically queried providers about specific situations in which TXA use could be indicated, which included obstetrical and gynecological situations, both acute and prophylactic use. Baseline differences in demographics and practice types was also elicited from the questionnaire. Questions regarding concerns and barriers to use of TXA was asked and providers could choose from multiple answers. The project was reviewed by the Institutional Review Board and approved.

\section{RESULTS}

There were 81 responses with a survey response rate of $27 \%$. The majority, $63 \%$, of responders were generalist, followed by providers who identified themselves as MFM 33\%. The majority of providers worked at an academic center $54 \%$, followed by those who practiced in a hospital-based setting $30 \%$, and then private practice $21 \%$. The majority of responders had been in practice $>20$ years $72 \%$, followed by physicians who had been in practice 3-5 years 10\% (Table 1). TXA was mostly used during PPH $72 \%$, followed by heavy cyclic bleeding $42 \%$, and was least used in benign surgeries $5 \%$ such as myomectomy and hysterectomy (Figure 1a). The most common use of TXA was during PPH, however the most selected frequency $33 \%$ of use by respondents was $<10 \%$ (Figure $1 \mathrm{~b}$ ).

Results show that when the hospital had TXA as part of its PPH protocol, $86 \%$ of providers used TXA for PPH. When TXA was not part of the hospitals PPH or the providers did not know if TXA was part of the hospital's protocol $60 \%$ of providers at those hospitals did not use TXA (Table 2). When asked how comfortable providers would be at incorporating TXA into their hospitals protocol, and overwhelming number $95 \%$ said very or somewhat comfortable (Figure 2).

The main concern physicians had with TXA was thromboembolic events, $48 \%$, followed by lack of familiarity $23 \%$ and that they prefer other agents for bleeding $24 \%$. $33 \%$ of responders had no concerns with using TXA. Other concerns included cost $18 \%$ and risk of hypersensitivity reaction to medication $4 \%$ (Figure 3 ).

\section{DISCUSSION}


This survey is the first to our knowledge that seeks to determine use of TXA in clinical practice, elicit concerns and comfortability with use among obstetricians and gynecologists. Although TXA was most often used during a PPH, $52 \%$ of responders were only utilizing it $<10 \%$ of the time or not at all. This finding reveals the unexpected underutilization of TXA during $\mathrm{PPH}^{8}$. Given the WHO recommendations regarding incorporation of TXA into the standard postpartum hemorrhage protocol for vaginal and cesarean sections ${ }^{9}$, we expected to see a higher utilization of TXA in standard practice for PPH. The main issues with TXA use found in this study are: concerns for thromboembolic events, lack of familiarity, and a preference for other agents. As discussed previously, in review of the current research, adverse events were not seen with use of TXA for PPH. TXA is both cost-effective and efficient.

This study illustrates an association between increase use of TXA when it is incorporated into a hospital protocol for PPH. When the hospital had TXA as part of its PPH protocol, $86 \%$ of providers used TXA for PPH. This finding suggests that to an individual provider, recognition of the safety and effectiveness of TXA is more impactful at the local level than it is on a global level. Plainly stated, individual hospital protocols carry more weight than the World Health Organization to an individual obstetrician in practice. Interestingly, although the major concern with TXA use was increased risk of a thromboembolic event, an overwhelming number of respondents would support incorporating TXA into their hospital's protocols. This finding is a little more elusive, but may suggest that physicians might feel an added element of safety when using a treatment that is perceived to be controversial if its use is supported by their own hospital.

The survey has several limitations. The response rate of $27 \%$ is low and may lead to unrecognized bias in the results. Also, there may be selection bias as the majority of respondents were generalist and MFM physicians, which may have skewed the results toward use during obstetrical settings. In order to not burden respondents, the survey was kept short with 10 questions; this may have led to challenges to ascertain practice patterns of TXA use. Although the questions were carefully selected, it may have been pertinent to include questions about what motivated use of TXA. It would be interesting to compare use before and after WOMAN trial. Furthermore, it would be interesting to determine if changing hospital protocols is necessary for increased use versus if increase use happens due to new data and large outside influence. One explanation for greater use during obstetrics than gynecology may be the WOMEN trial and recent WHO recommendations for use during $\mathrm{PPH}$.

Disclosure of Interest: No conflicts of interest or disclosures.

Contribution of authorship: RBD was instrumental in creating and distributing the survey as well as data collection and writing. ML played an active role in the development of the survey and the editing of the manuscript. SN, CH, LM assisted in the development of the survey and the data analysis. JG assisted in the writing of the manuscript and the editing.

Details of ethics approval: The project was reviewed by the Institutional Review Board and approved on 12/22/2017. The final approval was by Stephanie Sima Ortel at Loyola University Medical Center. Project LU\# 210587.

Funding: No funding was use for this project.

\section{References}

1. Callaghan, WM., Kuklina, EV., Berg, CJ. Trends in postpartum hemorrhage: United States, 19942006. Am J Obstet Gynecol 2010;202:353.e1-6

2. Ngwenya S. Postpartum hemorrhage: incidence, risk factors, and outcomes in a low-resource setting. Int J Womens Health. 2016;8:647-650. Published 2016 Nov 2.

3. Klebanoff, J., Marfori, C., Ingraham, C., Wu, C., \& Moawad, G. (2019). Applications of Tranexamic acid in benign gynecology. Current opinion in obstetrics \& gynecology

4. Dunn C.J., Goa K.L. Tranexamic acid. Drugs. 1999;57(6):1005-1032. http://link.springer.com/ 10.2165/00003495-199957060-00017

5. Roberts I, Shakur H, Coats T, Hunt B, Balogun E, Barnetson L, et al. The CRASH-2 trial: a 
randomised controlled trial and economic evaluation of the effects of tranexamic acid on death, vascular occlusive events and transfusion requirement in bleeding trauma patients. Health Technol Assess. 2013;17(10):1-79. doi: 10.3310/hta17100.

6. McCormack PL. Tranexamic acid: a review of its use in the treatment of hyperfibrinolysis. Drugs 2012; 72:585-617

7. Panteli, M., Pountos, I., \& Giannoudis, P. V. (2016). Pharmacological adjuncts to stop bleeding: options and effectiveness. European journal of trauma and emergency surgery : official publication of the European Trauma Society, 42(3), 303-310. doi:10.1007/s00068-015-0613-x

8. WOMAN Trial Collaborators. Effect of early tranexamic acid administration on mortality, hysterectomy, and other morbidities in women with post-partum haemorrhage (WOMAN): an international, randomised, double-blind, placebo-controlled trial [published correction appears in Lancet. 2017 May 27;389(10084):2104]. Lancet. 2017;389(10084):2105-2116. doi:10.1016/S0140-6736(17)30638-4

9. WHO recommendation on tranexamic acid for the treatment of postpartum haemorrhage.World Health Organization, Geneva2017

10. (Barber EL, Lundsberg LS, Belanger K, Pettker CM, Funai EF, Illuzzi JL. Indications contributing to the increasing cesarean delivery rate. Obstet Gynecol. 2011;118(1):29-38. doi:10.1097/AOG.0b013e31821e5f65

11. Healy TE, Knight PR. Obstetric Anaesthesia. Wylie and Churchill-Davidson's A Practice of Anaesthesia. 7 th ed. London: Arnold; 2003

12. Ekeroma AJ, Ansari A, Stirrat GM. Blood transfusion in obstetrics and gynaecology. Br J Obstet Gynaecol 1997;104:278-84

13. Retrieved from https://www.cdc.gov/nchs/fastats/delivery.htm January 20, 2017Content source: CDC/National Center for Health Statistics

14. Gai MY, Wu LF, Su QF, Tatsumoto K. Clinical observation of blood loss reduced by tranexamic acid during and after caesarian section: A multi-center, randomized trial. Eur J Obstet Gynecol Reprod Biol 2004;112:154-7.

15. Sekhavat L, Tabatabaii A, Dalili M, Farajkhoda T, Tafti AD. Efficacy of tranexamic acid in reducing blood loss after cesarean section. J Matern Fetal Neonatal Med 2009;22:72-5.

16. Gohel M, Patel P, Gupta A, Desai P. Efficacy of tranexamic acid in decreasing blood loss during and after caesarean section: A randomized case controlled prospective study. Obstet Gynaecol India 2007;57:227-30.

17. Callender, S. T., Warner, G. T., \& Cope, E. (1970). Treatment of menorrhagia with tranexamic acid. A double-blind trial. British medical journal, 4(5729), 214-216. doi:10.1136/bmj.4.5729.2

18. Lukes, A., Freeman, E., Van Drie, D., Baker, J., Adomako, T. Safety of tranexamic acid in women with heavy menstrual bleeding: an open-label extension study. Womens Health (Lond) 2011 Sep; 7(5): 591-598. doi: 10.2217/whe.11.55).

19. Wu JM, Wechter ME, Geller EJ, Nguyen TV, Visco AG. Hysterectomy rates in the United States, 2003. Obstet Gynecol. 2007;110:1091-5.

20. Topsoee, M., Settnes, A., Ottesen, B., Bergholt, T. A systematic review and meta-analysis of the effect of prophylactic tranexamic acid treatment in major benign uterine surgery. Int J Gynaecol Obstet. 2017 Feb; 136(2): 120-127. Published online 2016 Dec 9. doi: 10.1002/ijgo.12047

21. Shaaban, M. M., Ahmed, M. R., Farhan, R. E., \& Dardeer, H. H. (2016). Efficacy of Tranexamic Acid on Myomectomy-Associated Blood Loss in Patients With Multiple Myomas: A Randomized Controlled Clinical Trial. Reproductive Sciences, 23(7), 908-912.

22. Topsoee MF, Bergholt T, Ravn P, et al. Anti-hemorrhagic effect of prophylactic tranexamic acid in benign hysterectomy-a double-blinded randomized placebo-controlled trial. American Journal of Obstetrics and Gynecology. 2016 Jul;215(1):72.e1-8. DOI: 10.1016/j.ajog.2016.01.184.

23. Retrieved on April 16, 2020 from http://www.caog.org/. Central Association of Obstetricians and Gynecologists.

\section{Figure Legends}


Table 1 Demographics of providers.

Figure 1a Providers asked which specific situations do they use TXA in.

Figure 1b Providers asked to select the frequency in which they use TXA during postpartum hemorrhage.

Table 2. If TXA was part of the hospital protocol for postpartum hemorrhage treatment, increase use was seen among providers.

Figure 2. Providers have a favorable view of incorporating TXA into hospitals protocols.

Figure 3. Providers selected concerns they had with TXA use.

\section{Hosted file}

Table 1. .docx available at https://authorea.com/users/324367/articles/452586-the-use-oftranexamic-acid-txa-among-obstetricians-and-gynecologists-a-pilot-study-of-currentclinical-practice

\section{Hosted file}

Figure 1. .docx available at https://authorea.com/users/324367/articles/452586-the-useof-tranexamic-acid-txa-among-obstetricians-and-gynecologists-a-pilot-study-of-currentclinical-practice

\section{Hosted file}

Table 2. .docx available at https://authorea.com/users/324367/articles/452586-the-use-oftranexamic-acid-txa-among-obstetricians-and-gynecologists-a-pilot-study-of-currentclinical-practice

\section{Hosted file}

Figure 2..docx available at https://authorea.com/users/324367/articles/452586-the-use-oftranexamic-acid-txa-among-obstetricians-and-gynecologists-a-pilot-study-of-currentclinical-practice

\section{Hosted file}

Figure 3.docx available at https://authorea.com/users/324367/articles/452586-the-use-oftranexamic-acid-txa-among-obstetricians-and-gynecologists-a-pilot-study-of-currentclinical-practice 